\title{
Laryngeal Ultrasound for Assessment of Supraglottic Laryngitis with Abscess
}

\author{
Po-Han Chiu, Chun-Wen Shih, Yih-Jeng Tsai, Rayleigh Ping-Ying Chiang
}

\begin{abstract}
Here we report a rare case, for this era, of supraglottic laryngitis with abscess formation after antibiotics use. Laryngeal ultrasound for abscess detection and follow-up has advantages over neck computed tomography. This modality can assess vocal cord movement noninvasively with the benefits of cost effectiveness, easy availability, freedom from radiation, and high resolution-factors that are superior to other modalities. Although laryngeal abscesses are rare, clinicians should be aware of their myriad presentations. Acute supraglottitis with abscess formation may cause potentially fatal obstruction of the airway. Early recognition and intervention to prevent morbidity and mortality is thus very important.
\end{abstract}

Keywords: Laryngeal abscess, Laryngeal ultrasound, Supraglottic laryngitis.

How to cite this article: Chiu PH, Shih CW, Tsai YJ , Chiang RPY. Laryngeal Ultrasound for Assessment of Supraglottic Laryngitis with Abscess. Int J Phonosurg Laryngol 2013;3(1): 24-27.

\section{Source of support $\mathrm{Nil}$}

Conflict of interest: None declared

\section{INTRODUCTION}

Laryngeal abscess formation has become rare after the advent of antibiotics in the 1930s and 1940s; it was once a frequent complication of systemic infections such as typhoid fever, and it was less frequently caused by measles, scarlet fever, erysipelas, tuberculosis, and so on. ${ }^{1}$ A cute laryngeal abscess formation has been associated with three causes in the present era: M alignancy, airway instrumentation, and a pre-existing laryngocele. ${ }^{1}$ Fiberscopy of the larynx and computed tomography $(\mathrm{CT})$ neck with contrast are the commonest ways to evaluate and diagnose laryngeal abscesses. In addition, ultrasound represents an alternative modality to evaluate laryngeal abscess formation and assess vocal cord movement noninvasively. The larynx is an aircontaining structure with thyroid cartilage as an outer element. $M$ any of its components lie superficially in the neck and have good inherent soft tissue contrast. ${ }^{2} \mathrm{High}$ resolution ultrasound is suitable for examination of the larynx, and it is low cost and radiation free. Thus, this modality may provide an alternative means for evaluation of laryngeal abscess formation.

\section{CASE REPORT}

A 59-year-old man, who had uncontrolled type II diabetes mellitus, was presented with a rapidly progressing sore throat, odynophagia, and dysphagia within a 2-day time frame. The patient was sent to our emergency department due to stridor with shortness of breath. There was no history of trauma, recent operations of the head and neck, or swallowing of a foreign body. The patient was febrile $\left(38.5^{\circ} \mathrm{C}\right)$ and had a muffled voice. The results of physical examination showed left cervical lymphadenopathy over levels II and III. Neck soft tissue X-ray was performed first and the 'thumb sign' was detected. Fiberscopy of the larynx was used directly to evaluate the larynx, and this revealed injection and swelling of the epiglottis with impending airway obstruction. Immediately, nasoendotracheal intubation was performed under laryngoscopic guidance. Leukocytosis $(14,000 / \mu \mathrm{l})$ with a neutrophil predominance (87.0\%) was noted. Intravenous antibiotics of amoxicillin/ clavulanic acid were given. Blood sugar was controlled by insulin under the guidance of an endocrinologist. During hospitalization, laryngoscopy revealed decreased swelling of the epiglottis, and the nasoendotracheal tube was extubated 2 days after admission. Blood cultures were negative. Fiberscopy of the larynx demonstrated limited left vocal fold movement and swelling of the left aryepiglottic fold and ventricular fold (Fig. 1). A bscess formation was suspected. Neck CT with/without contrast was arranged, and this revealed laryngeal abscess formation (Figs 2 and 3 ). L aryngeal ultrasound was also arranged, and this showed a hypoechoic and heterogeneous lesion at the left paraglottic space with limited left vocal cord movement (Fig. 4). Incision and drainage was suggested initially, but the patient

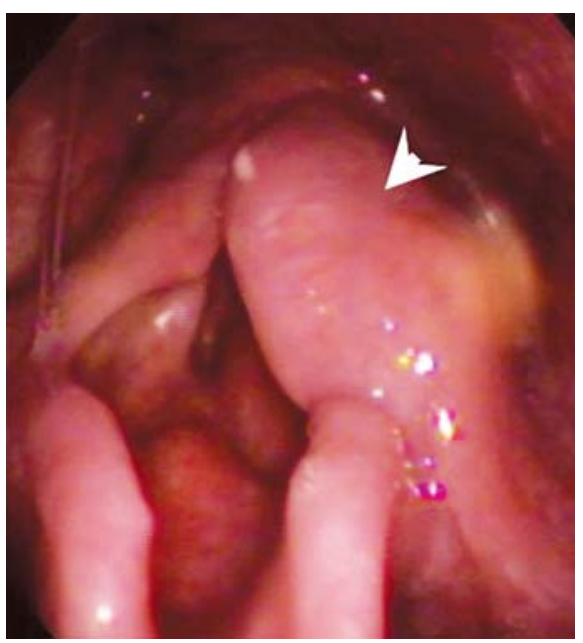

Fig. 1: Laryngoscopy showing swelling of the left aryepiglottic fold with suspected abscess formation 


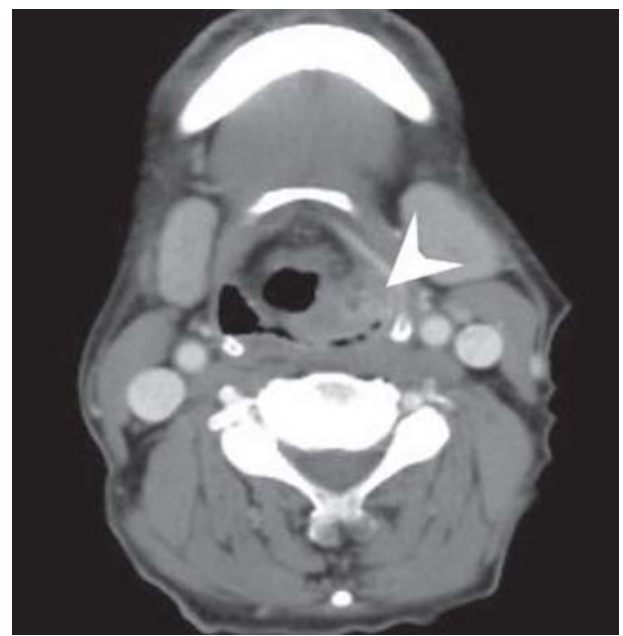

Fig. 2: Axial view of neck CT with contrast showing hypodensity with rim enhancement consistent with paraglottic abscess formation (arrow head)

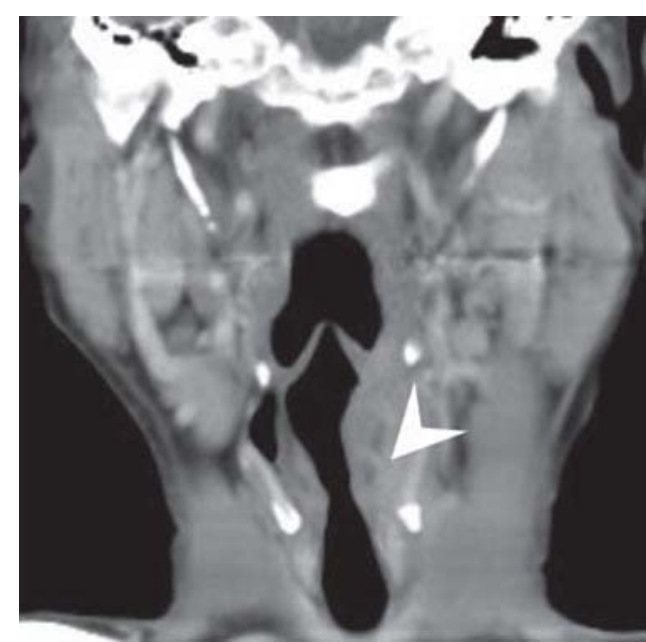

Fig. 3: Coronal view of neck CT with contrast showing hypodensity with rim enhancement consistent with paraglottic abscess formation (arrow head)

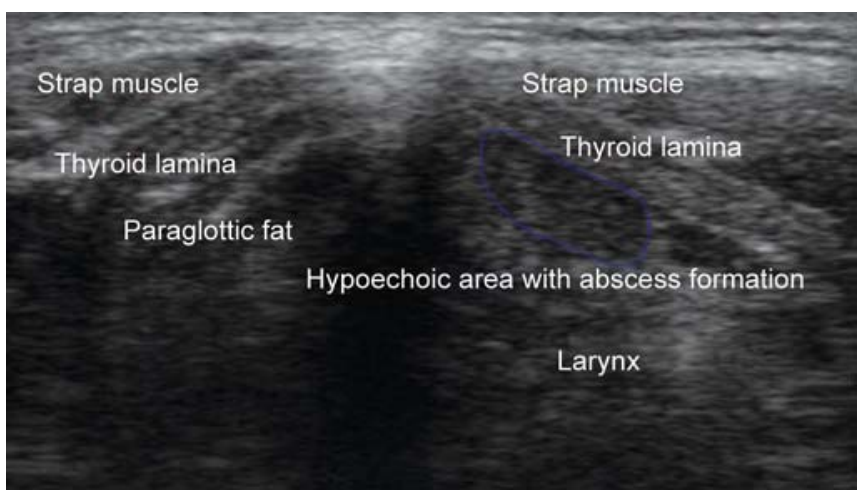

Fig. 4: Laryngeal ultrasound showing a left paraglottic hypoechoic area with paraglottic abscess and loss of paraglottic fat (encircled by blue line)

hesitated and preferred medical treatment. A fter intravenous antibiotics of amoxicillin/clavulanic acid for 2 weeks, laryngoscopy showed diminished swelling of the left aryepiglottic fold and ventricular fold (Fig. 5). Follow-up

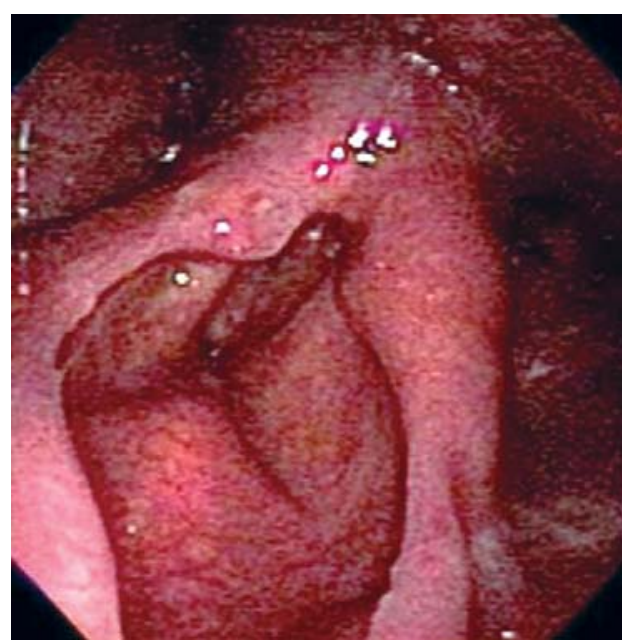

Fig. 5: Laryngoscopy showing diminished swelling of the left aryepiglottic fold and no limitation of vocal cord movement 2 weeks later

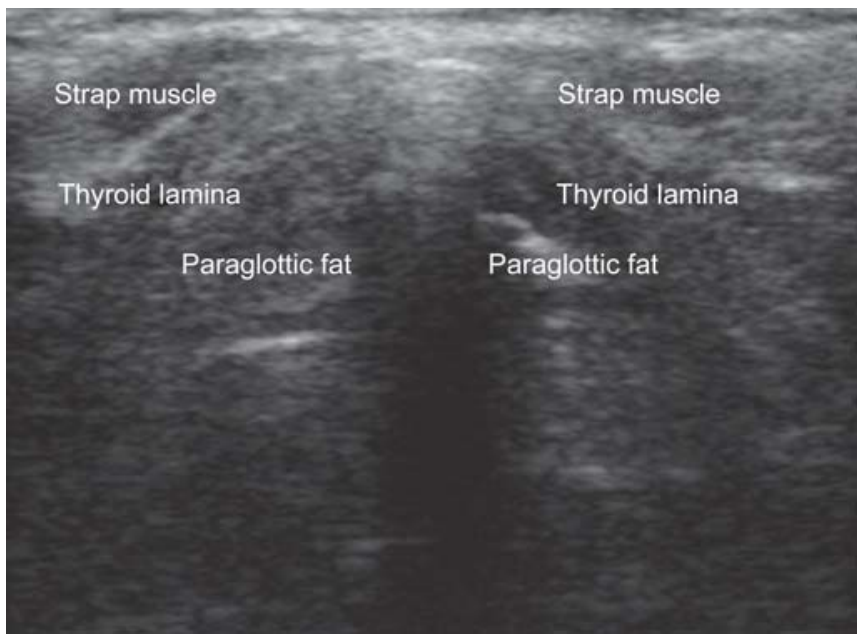

Fig. 6: Laryngeal ultrasound showing symmetrical laryngeal structure with good vocal cord movement

laryngeal ultrasound also demonstrated resolution of the paraglottic abscess with no vocal cord movement limitations (Fig. 6). The patient was discharged and presented no further symptoms or signs after 3 months of follow-up.

\section{DISCUSSION}

A cute supraglottitis and epiglottitis may cause potentially fatal obstruction of the airway, ${ }^{3}$ but early diagnosis and management may reduce morbidity and mortality rates. ${ }^{4,5}$ The incidence of abscess formation associated with supraglottitis is unknown, and this combination has rarely been reported. It is unclear what factors play the most important roles in abscess formation in the neck. ${ }^{6} \mathrm{~A}$ preexisting epiglottic cyst might be associated with a suppurative infection of the supraglottis. ${ }^{7}$ One 17 -year-old boy presented with aryepiglottic abscess formation that manifested as epiglottitis in 2009. ${ }^{8}$ The clinical presentation 
was similar to our case, but the treatment and evaluation were quite different. The clinical implication is that clinicians should be aware of the myriad presentations and unusual causative organisms of laryngeal abscesses even in this era of widespread immunization with the Haemophilus influenzae $B$ ( $\mathrm{HiB}$ ) vaccine. ${ }^{8}$ In the preantibiotic era, laryngeal abscesses were a relatively common problem, and carried a mortality rate approaching $100 \%$ until the importance of early drainage and tracheostomy was recognized; however, it still remains a highly dangerous disease, and, depending on its location, may result in serious permanent deficits. A fter the widespread use of penicillin in the mid-1940s, laryngeal abscesses became an uncommon problem, and they are now relatively rare.

Our case presented with supraglottic laryngitis complicated with airway obstruction, necessitating immediate intubation. The abscess was recognized after extubation. Recognition of paraglottic abscess by laryngoscopy was difficult even without endotracheal tube interference. Neck CT with contrast demonstrated hypodensity with rim enhancement consistent with a paraglottic abscess. L aryngeal ultrasound showed a paraglottic hypoechoic area between the thyroid cartilage and paraglottic fat. I mpairment of left vocal cord movement could be confirmed dynamically. Considering the cost and radiation exposure, the utility of laryngeal ultrasound has advantages, but using it makes it hard to differentiate tumors or abscesses simply based on neck ultrasonography because these lesions both present as ill-defined hypoechoic lesions. Limitations of laryngeal ultrasound might be resolved by overall evaluation of the clinical picture and expert technique for performing the ultrasound.

Ultrasound has no radiation and is easily available. In this case, except for fiberscopy of the larynx, ultrasound was used for follow-up-effectively replacing neck CT. Needle drainage of the abscess can also be done under ultrasound guidance. Evaluation of abscess extension is sometimes hard under fiberscopy, especially when the paraglottic space and pre-epiglottic space are involved; how ever, ultrasonography can help the clinician to evaluate and follow-up such extensions.

Thus, laryngeal ultrasound is a valuable tool for the otolaryngologist. It is a real-time procedure and can offer higher resolution than $\mathrm{CT}$ and magnetic resonance imaging (MRI) because of the superficial location of the larynx. L aryngeal ultrasound is also important in assessments of laryngeal squamous cell carcinoma staging, anterior cervical mass, vocal cord function, laryngocele/cysts, dysphagia, epiglottitis, fetal airways, infantile stridor (congenital cysts), subglottic hemangioma, and laryngeal stenosis (stenting). ${ }^{9}$

Scanning of the larynx in our case was done through the cricothyroid region angling upward. The thyroid laminae were hypoechoic inside the strap muscle and the paraglottic fat was hyperechoic due to fatty connective tissue. The left side of the paraglottic space was replaced by a hypoechoic, ill-defined area consistent with abscess formation. The abscess was limited to the paraglottic space without extension to the pre-epiglottic space.

The limitations of laryngeal ultrasound include interference with calcified cartilage due to old age, disturbance by air in the upper aerodigestive tract, and its dependency on operator experience. N evertheless, the utility of laryngeal ultrasound in acute supraglottic laryngitis offers many advantages. Rapid screening and early detection may prevent morbidity and hasten interventions.

\section{CONCLUSION}

Though the incidence of laryngeal abscess formation has been decreased with antibiotics use, early recognition and intervention to prevent morbidity or mortality remain very important. Laryngeal ultrasound for acute supraglottic laryngitis was able to detect abscess formation and also evaluate movement of the vocal cord. In combination with laryngoscopy, ultrasound provides a radiation free and low cost al ternative to neck $\mathrm{CT}$.

\section{REFERENCES}

1. Canalis RF, J enkins HA, Osguthorpe JD. A cute laryngeal abscesses. A nn Otol Rhinol Laryngol 1979;88:275-79.

2. L oveday E. The larynx. In: A huja, Evans R (editors). Practical head and neck ultrasound. New Y ork: Cambridge University Press; 2007:107-17.

3. Hafidh MA, Sheahan P, Keogh I, McConn Walsh R. A cute epiglottitis in adults: A recent experience with 10 cases. J L aryngol Otol 2006;120:310-13.

4. Ossoff RH, W olf AP, Ballenger J]. A cute epiglottitis in adults: Experience with 15 cases. Laryngoscope 1980;90:1155-61.

5. F rantz TD, Rasgon BM, Quesenberry CP. A cute epiglottitis in adults: A nalysis of 129 cases. J AM A 1994;272:1358-60.

6. Gillett D, Eynon-L ewis NJ . Supraglottitis and abscess formation. J Laryngol Otol 2011 Jan;125(1):99-102.

7. Y oon TM , Choi JO, Lim SC, Lee JK. The incidence of epiglottic cysts in a cohort of adul ts with acute epigl ottitis. Clin Otolaryngol 2010 Feb;35(1):18-24.

8. Reed J, Shah RK, Jantausch B, Choi SS. A ryepiglottic abscess manifesting as epiglottitis. A rch Otolaryngol Head N eck Surg 2009 Sep;135(9):953-55.

9. B eale TJ, Rubin JS. L aryngeal ultrasonography. In: Orloff LA . Head and neck ultrasonography. San Diego, California: Plural Publishing Inc.; 2008:183-202. 


\section{ABOUT THE AUTHORS}

\section{Po-Han Chiu}

Department of Otolaryngology, Keelung Hospital, Department of Health, Executive $Y$ uan, Taiwan (ROC)

\section{Chun-Wen Shih}

Department of Otolaryngology, Shin Kong W u Ho-Su M emorial Hospital, Taipei, Taiwan

\section{Yih-Jeng Tsai}

Department of Otolaryngology, Shin Kong Wu Ho-Su M emorial Hospital; Department of Otolaryngology, Head and Neck Surgery School of M edicine, Fu Jen Catholic University, Taipei, Taiwan

\section{Rayleigh Ping-Ying Chiang}

Department of Otolaryngology, Head and Neck Surgery and Sleep Center, Taipei Veterans General Hospital, Taipei, Taiwan; Department of Otolaryngology, Head and Neck Surgery, School of Medicine, Taipei M edical University, Taipei, Taiwan; Sleep Technology Special Interest Group, INSIGHT Center, National Taiwan University, Taipei, Taiwan

CorrespondenceAddress: D epartment of Otolaryngology, Head and N eck Surgery and Sleep Center, Taipei V eterans, General Hospital No. 201, Sec. 2, Shipai Rd, Beitou, Taipei-11217, Taiwan (ROC) e-mail: rayleighchiang@ ntu.edu.tw 